A N N A L E S Annales de Bretagne et des Pays de l'Ouest

\title{
Les Annales briochines, 1771, Saint-Brieuc : histoire d'une ville et d'un diocèse
}

\section{Philippe Loupès}

\section{(2) OpenEdition}

1 Journals

Édition électronique

URL : http://journals.openedition.org/abpo/2803

DOI : $10.4000 /$ abpo.2803

ISBN : 978-2-7535-3513-8

ISSN : 2108-6443

Éditeur

Presses universitaires de Rennes

Édition imprimée

Date de publication : 30 juin 2014

ISBN : 978-2-7535-3511-4

ISSN : 0399-0826

Référence électronique

Philippe Loupès, "Les Annales briochines, 1771, Saint-Brieuc : histoire d'une ville et d'un diocèse ", Annales de Bretagne et des Pays de l'Ouest [En ligne], 121-2 | 2014, mis en ligne le 30 juin 2014, consulté le 22 septembre 2020. URL : http://journals.openedition.org/abpo/2803 ; DOI : https://doi.org/ $10.4000 / a b p o .2803$ 
social et, à l'intérieur de la compagnie, le maintien des hiérarchies. Son idéal? Celui du " très bon chanoine, bien exact à tous ses devoirs (p. 426) "... tout en restant, à l'occasion, sensible aux honneurs : dire la messe, porter les objets du culte pendant les processions...

Enfin, à partir de 1789, Nepveu apparaît comme un chanoine hostile au nouveau cours des choses mais manquant singulièrement de clairvoyance. Tout comme les chapitres à travers le royaume continuent à enregistrer le train-train capitulaire dans leurs registres, Nepveu consigne les événements habituels affectant sa compagnie - nomination d'un archidiacre, procession de la Fête-Dieu, décès d'un confrère... - et nourrit son carnet mondain - on ne compte plus les naissances, mariages ou décès. La pose des scellés à la cathédrale le 4 décembre 1790 marque certainement une rupture : dès lors, on sent une certaine irritation poindre. Après le serment de 1791, il refuse de croire "que les Cures de Ville qui, suivant le Décret, sont déclarées vacantes, soient remplies par ces mauvais sujets (p. 507) ", c'està-dire par les jureurs. Plus tard, il se laisse aller à un commentaire acerbe lors de l'élection de l'évêque constitutionnel du Mans. Puis, quand ce dernier ordonne ses premiers prêtres, il les qualifie de "rebut de l'ancien séminaire (p. 520) ". A-t-il pour autant compris que le royaume était entré dans une nouvelle ère? On en doute. Les États généraux ne valent que parce que " touttes les Compaignies y étoient, comme d'usage (p. 471) ", la prise de la Bastille et la Grande peur ne suscitent qu'un bref commentaire et un renvoi laconique " aux papiers publics de ce tems (p. 476)", l'abolition des privilèges le laisse de marbre (" On n'a jamais vu chanter un Te Deum pour si peu de chose ", p. 481). Plus tard, après bien des péripéties, il devient chanoine honoraire du chapitre concordataire mais " ce n'est plus comme autrefois (p. 613)" et il donne l'impression d'être un peu perdu dans un monde que n'est plus tout à fait le sien.

En définitive, nous tenons là un ouvrage qui est beaucoup plus que l'édition d'un écrit du for privé, un journal qui est beaucoup plus qu'un document local. Certes, à l'échelle mancelle, il permet de pallier l'absence des registres de délibérations du chapitre cathédral et dévoile de l'intérieur le fonctionnement de certains aspects de la complexe machinerie capitulaire. Mais, plus largement, il s'agit d'un travail de référence pour tous ceux qu'intéresse le monde des chanoines. L'imposante introduction scientifique (p. 13-87) non seulement dégage très clairement les centres d'intérêt du Journal mais constitue un véritable essai sur l'univers capitulaire, certes au prisme de l'expérience de Nepveu et du Mans... mais qui vaut pour bien d'autres chanoines et chapitres. Les références bibliographiques livrées en fin de volume ou en notes dans l'introduction permettent quant à elles de guider l'entrée parfois délicate dans le monde des chanoines. À cela, ajoutons le très complet glossaire de la vie canoniale (p. 657-679) appelé à devenir un instrument de travail essentiel et pratique puisque renvoyant systématiquement au texte de Nepveu.

Olivier CHARLES

RufFelet, Christophe-Michel, Les Annales briochines, 1771, Saint-Brieuc : histoire d'une ville et d'un diocèse, CHARLES, Olivier (éd. et dir.), Rennes/Saint-Brieuc, PUR/ Société d'émulation des Côtes-d'Armor, coll. " Mémoire commune ", Rennes, 2013, 625 p.

Né à Saint-Brieuc dans la bonne bourgeoisie cultivée de fonction et de commerce, Christophe-Michel Ruffelet fait ses études au collège de sa ville. Peuplée de 
6000 habitants, Saint-Brieuc est alors un siège épiscopal relativement médiocre, comme il y en a tant dans la France d'Ancien Régime, une cité qui se signale par une forte densité ecclésiastique. Ordonné prêtre en 1749, Ruffelet fréquente la boutique de Prud'homme, l'imprimeur et libraire briochin bien connu, et commence à rassembler sa belle bibliothèque, qui sera la plus importante de Bretagne, après celle du président Robien. Entré au chapitre collégial Saint-Guillaume en 1771, il n'accède au chapitre cathédral que trop tard (le 17 juillet 1789!); quelques mois plus tard, tous les chapitres de France sans exception sont éteints. Depuis longtemps, il aspirait à cette consécration; avec son cas, on a la preuve des nombreuses démarches et tractations pour accéder à un canonicat, qu'on ne fait généralement que subodorer. Emprisonné sous la Terreur, il récupère lors de son élargissement sa chère bibliothèque avec les fonctions de gardien de cette dernière. Cette collection de quelque 4000 ouvrages que l'inventaire révolutionnaire dressé dans l'été 1794 permet d'appréhender est au centre de sa vie, comme elle est au cœur de sa demeure. Elle est tout à la fois bibliothèque, cabinet de curiosité, cabinet scientifique. Logiquement assise sur une base religieuse, elle est cependant diversifiée et largement ouverte sur le monde contemporain. En 1803, Ruffelet réintègre le nouveau chapitre concordataire, mais meurt peu après, en 1806, léguant tous ses livres à l'évêque de Saint-Brieuc pour " être employés à l'instruction des séminaristes ". Ces milliers de volumes ont effectivement alimenté la bibliothèque de séminaire et la bibliothèque des Archives départementales.

La bibliothèque du chanoine avait été précocement le socle de ses investigations qui avaient abouti en 1771 à la publication à Saint-Brieuc de ses Annales, petit livre broché de quelque 250 pages, au prix abordable de 25 sols. Ces annales sont à vrai dire un curieux livre, constitué de plusieurs textes juxtaposés annonçant un ouvrage plus important qui ne verra jamais le jour. Après une description liminaire de son diocèse, Ruffelet se laisse porter par la chronologie, de l'Antiquité jusqu'en 1768. Il embrasse donc largement, des lointaines origines de la Bretagne jusqu'à " l'affaire de Bretagne " au service de laquelle il engage sa plume. Suivent dans l'esprit érudit de l'époque, des notices diverses, comme une liste documentée des paroisses du diocèse, la chronologie des évêques de Saint-Brieuc assortie de véritables biographies. La formule typée des annales égrène un bref récit année par année, les notices s'étoffant à l'époque moderne avec une information plus abondante et plus sûre. Pour le Moyen Âge, Ruffelet retient les épiscopats, les fondations religieuses, les faits d'armes ou bien des événements marquant pour l'histoire de la Bretagne comme cette famine de 1161 où les hommes furent "obligés à manger jusqu'à la terre " (p. 232). À l'époque moderne, son attention est plus spécialement retenue par les nombreuses fondations de la Réforme catholique et aussi les séquelles des guerres de Religion, comme le pillage de Saint-Brieuc en 1592 par l'armée des Espagnols et des Lorrains. Sur la longue durée pluriséculaire, le passionne l'histoire du duchépairie de Penthièvre dont " l'histoire est trop intéressante, trop intimement liée à l'histoire briochine " pour ne pas en faire un long tableau.

Ce seul texte en lui-même valait la publication et l'on peut remercier la Société d'émulation des Côtes-d'Armor d'avoir joué son vrai rôle de société savante en rendant un document de première importance facilement accessible aux lecteurs curieux ou érudits et à la communauté des chercheurs. Mais le présent ouvrage dépasse largement ce niveau, avec sa structure emboîtée. Non seulement y sont adjoints des notes, des commentaires, des analyses, des annexes le rendant parfaitement intelligible, mais encore, à un niveau supérieur, des études replaçant le tout dans l'historiographie contemporaine. L'ensemble est impressionnant par son ampleur, sa pertinence, son érudition qui est en prise avec les plus récents travaux. 
Pour mener à bien cette tâche ambitieuse, le maître d'œuvre, Olivier Charles, expert en études capitulaires, s'est adjoint une quinzaine de collaborateurs dont les compétences complémentaires " couvrent " la totalité de l'œuvre, jeunes chercheurs prometteurs et historiens confirmés. Les angles d'approche variés donnent du relief à l'ensemble. Impossible d'énumérer toutes les contributions : la moisson est impressionnante, allant bien au-delà de ce que l'on est en droit d'attendre pour la bonne intelligence d'un document. À titre d'exemple, on sait tout, ou presque, sur le Saint-Brieuc du chanoine Ruffelet, jusqu'au climat frais qui y régnait, grâce aux relevés météorologiques effectués entre 1772 et 1790 par Jean-Louis Bagot, docteur en médecine et maire de la ville. Parmi toutes ces contributions de qualité, la plus importante est sans doute celle d'Oliver Charles intitulée "Les chanoines historiens en France aux XVII et XVII e siècles ". Remarquablement informée et problématisée, c'est le point actuel sur cette question. Deux cartes suggestives y montrent que l'histoire capitulaire est le fait de la France septentrionale, Bretagne exceptée!

En historiens critiques aux bons sens du terme, les co-auteurs de cet ouvrage n'ont jamais pour Ruffelet la complaisance coupable, fréquente chez les biographes. Par exemple, pour son analyse de la société rurale, Yann Lagadec ose même dans son titre le terme fort des " œillères du chanoine Ruffelet ", lequel il est vrai se borne à des stéréotypes et ne voit guère les paysans. Curieusement, Ruffelet reste également muet sur les querelles religieuses sous l'Ancien Régime : rien sur le protestantisme, sur le jansénisme, sur les dévotions collectives... En fait, ces lacunes ne minimisent pas l'intérêt des Annales qui sont pour l'essentiel la construction d'une identité diocésaine. Dans ce domaine, Ruffelet n'est certes pas un pionnier; il s'inscrit dans une longue lignée de chanoines érudits comme Hiérosme Lopès à Bordeaux ou du célèbre abbé Lebeuf pour Auxerre puis Paris. Lui aussi veut honorer sa " patrie ", comme il le dit à plusieurs reprises, d'autant que son diocèse pâtit en Bretagne d'un déficit de notoriété. Ses annales sont porteuses d'un projet cohérent de "recharge identitaire ", prometteur aussi pour l'avenir du diocèse. Cela dit, Ruffelet n'innove en rien; il se conforme à la tradition pour laquelle l'histoire d'un diocèse demeure dans sa structure celle de ses évêques. Mais sur cette trame classique, les questions de fond ne sont pas éludées, comme les origines de la ville avec son saint éponyme. Ruffelet mentionne les indécisions autour de saint Brieuc ( $\mathrm{V}^{\mathrm{e}}$ ou $\mathrm{VI}^{\mathrm{e}}$ siècle?), il ne masque pas le "trou béant " entre saint Brieuc et le premier de ses successeurs à être attesté en 1032. C'est d'ailleurs, avec compétence qu'il se passionne pour l'histoire de l'Antiquité; plus de $10 \%$ des ouvrages sa bibliothèque ne relèvent-ils pas de cette période?

La France de l'Ouest s'est engagée résolument dans la publication de mémoires d'ecclésiastiques, comme Les Souvenirs d'un nonagénaire, en l'occurrence FrançoisYves Besnard, ou les Mémoires du chanoine Le Sage, toutes sources passionnantes. Certes, on ne saurait attendre des Annales Briochines des "tranches de vie ", comme on en trouve dans Le livre de raison de Jean-Gaspard de Grasse, chanoine de Cavaillon (éd. aux éditions du CTHS en 2002), ou le Journal du chanoine du Mans, René-Pierre Nepveu de La Manouillère (édité aux PUR en 2013); c'est simplement affaire de genre : les annales sont un exercice d'érudition, non propice aux faits divers souvent anodins ou aux épanchements.

Cet homme de la République des Lettres méritait de sortir de l'oubli, comme d'ailleurs l'ensemble de l'ordo canonicus longtemps négligé par la recherche historique, en fonction d'anachroniques jugements de valeur qui n'ont pas leur place en histoire. Le chanoine Ruffelet fut un polygraphe dont malheureusement sont perdus la plupart des écrits. Polygraphe, il l'était dans sa manière de travailler, honnête, méticuleuse, quasi mauriste; ses sources furent sans doute les archives de la collégiale Saint-Guillaume, sa bibliothèque et un réseau érudit difficile à cerner. Ses 
Annales briochines, tout à la fois riches et lacunaires, sont une source documentaire de premier plan, non seulement pour Saint-Brieuc, mais aussi pour la Bretagne et l'histoire en général, en particulier urbaine et religieuse.

Philippe LoupÈs

GOUBERT, Jean-Pierre, Malades et médecins à Saint-Malo à la veille de la Révolution, Rennes, PUR, coll. « Histoire ", 2013, 166 p.

Jean-Pierre Goubert considère " la première médicalisation " des années 17701780 en souhaitant mettre en valeur les originalités de la ville de Saint-Malo et de son " pays " le Clos-Poulet, la réponse d'une société face à la maladie et à la mort, ainsi que " le profond écart qui sépare la société locale de l'époque par rapport à notre début de XXI e siècle " (p. 11). Diverses sources, institutionnelles et médicales, sont mobilisées : archives hospitalières, dossiers sur les maladies épidémiques et les pauvres malades de la subdélégation de Saint-Malo, enquêtes sur le corps médical, les secours distribués ou topographies médicales locales. En revanche, la voix des malades demeure inaudible : livres de recettes, correspondances privées ou mémoires ne sont pas sollicités. En complément des documents d'archives, l'auteur s'appuie sur des études locales datant principalement du XIX ${ }^{\mathrm{e}}$ siècle et de la première moitié du $\mathrm{Xx}^{\mathrm{e}}$ siècle. De manière générale, les références historiographiques ne sont pas mentionnées, si ce n'est dans une bibliographie finale faisant une large place à l'historiographie française des années 1960-1980. L'impasse faite sur l'abondante bibliographie anglo-saxonne, venue notamment documenter depuis les années 1980 les modalités de la relation thérapeutique, nuit à la prise en compte des renouvellements historiographiques récents. La faible sollicitation des recherches francophones menées depuis les années 1990 empêche également Jean-Pierre Goubert de mettre en évidence les particularités malouines par rapport au cadre breton et national. Soulignons également que, tout en se défendant de moquer les représentations et pratiques du passé, l'auteur délivre une lecture positiviste, en opposant une pensée " scientifique " contemporaine (sans la définir pour autant) à des méthodes et travaux modernes " préscientifiques" (p. 15, 84, 104, 137).

Les deux premières parties de l'ouvrage, consacrées aux secours n'échappent pas à l'opposition entre " foi, croyances, coutumes " d'un côté (partie 1) et médecine " savante » de l'autre (partie 2), faisant fi des liens susceptibles d'unir ces pratiques : les pauvres malades rejoignant les institutions charitables ne sont-ils pas soignés aussi bien par les religieux que par les élites médicales de la ville? Les formations, pratiques de soins et rémunérations variées des soignants non " savants " (notables, religieux, dentistes et arracheurs de dents, matrones, oculistes, bandagistes, herniaires, rebouteux, renoueurs, remancheurs, rhabilleurs et autres guérisseurs) ne sont pas distinguées pour expliquer les choix thérapeutiques des malades. Les catégorisations sont rarement définies, à l'image des " charlatans " mêlant soignants empiriques, itinérants et même religieuses hospitalières! Au final, les classifications proposées semblent peu convaincantes et représentatives du point de vue des praticiens " savants " en lutte contre les autres recours, que l'auteur se défend pourtant d'adopter. En outre, ce dernier ne précise pas les raisons qui poussent les malades à choisir tel ou tel thérapeute, postulant que le premier recours est celui de la religion et des soignants " traditionnels ", la sollicitation des médecins et chirurgiens arrivant en second lieu, ce qui demeure contestable pour une frange non négligeable des malades de la période prérévolutionnaire. 